\title{
Effects of Removing the Adventitia on the Mechanical Properties of Ovine Femoral Arteries In Vivo and In Vitro
}

\author{
Edmundo Cabrera Fischer, $\mathrm{PhD}^{*, \dagger}$; Daniel Bia Santana, PhD**; Yanina Zócalo, MD, PhD**; \\ Juan Camus*,†; Eduardo de Forteza*; Ricardo Armentano, PhD*,**
}

\begin{abstract}
Background: The aims were to characterize in muscular arteries (a) the passive and active effects of the adventitia on vessel biomechanical properties and conduit function (CF), and (b) potential differences between the adventitial role in elastic and muscular arteries.

Methods and Results: Ovine femoral arteries were studied in vivo and in vitro (reduced smooth muscle-tone) in a circulation mock-up during hemodynamic conditions similar to those found in vivo. Pressure and diameter were assessed before and after removing the adventitia. The arterial compliance, distensibility, stiffness $\beta$-index and CF were quantified. Results were compared with those obtained in brachiocephalic trunks. In vivo, after removing the adventitia there was a nonsignificant diameter reduction and an increase in stiffness $(P<0.05)$. The CF decreased in the early recordings $(P<0.02)$. In vitro, there were no biomechanical changes but vascular dilatation after the adventitia removal. Biomechanical changes associated with the adventitia removal were higher in muscular arteries, whereas diameter changes were major in elastic vessels.
\end{abstract}

Conclusions: After removing the adventitia, (a) the arterial stiffness and CF were modified in vivo only, suggesting the changes could be ascribed to variations in smooth muscle tone, and (b) changes in elastic and muscular arteries were quantitatively different. (Circ $J$ 2010; 74: 1014-1022)

Key Words: Adventitia; Arterial conduit function; Arterial stiffness; Elastic arteries; Muscular arteries

$\mathbf{T}$ he arterial adventitia is classically considered a physical barrier separating tissues, a passive structural support and a scaffold for nerve endings and the vasa vasorum. ${ }^{1}$ However, recent findings suggest that the adventitia is an active layer that could play an important role in the physiological regulation of arterial smooth muscle tone, ${ }^{2-5}$ modulating the response to stimuli such as noradrenalin and angiotensin II, and regulating nitric oxide release from the endothelium, thus counterbalancing the vasoconstrictor effects on smooth muscle cells. ${ }^{6}$ An active role of the adventitia in determining arterial properties has been proposed in pathological states such as atherosclerosis ${ }^{7}$ and systemic hypertension, ${ }^{8}$ and in arterial wall remodeling. ${ }^{2,9,10}$

\section{Editorial p 854}

Recently, we demonstrated in vivo that the adventitia of central elastic arteries in animals has a regulatory function in arterial wall dynamics and that its removal determines changes in arterial viscoelasticity, buffering and conduit functions (CFs), ${ }^{4}$ and in the left ventricle dynamic afterload determinants, ${ }^{3}$ through a smooth muscle-related active mechanism that was not found in the in vitro experiments. Our study had the relevance of characterizing, for the first time, the adventitia's role in central elastic arteries studied in vivo. ${ }^{4}$ As is known, central elastic and peripheral muscular arteries play different roles in the arterial system and have different biomechanical and structural properties. For instance, compared with central elastic arteries, the peripheral vessels have less compliance and higher viscosity. ${ }^{11,12}$ Therefore, storage capability would be concentrated in the elastic arteries, whereas the dissipative capability (mainly exerted by smooth muscle cells) would be localized mainly in the peripheral muscular arteries.11,12 Additionally, the greater amount of smooth muscle in peripheral arteries would determine a higher capability of actively controlling the arterial diameter, wall biomechanics, and so the impedance to blood flow. Hence, the adventitial effects on arterial biomechanics

Received July 9, 2009; revised manuscript received January 4, 2010; accepted January 12, 2010; released online March 30, 2010 Time for primary review: 39 days

*Favaloro University, Buenos Aires, Argentina, **Physiology Department, School of Medicine, Republic University, Montevideo, Uruguay, ${ }^{\dagger}$ Member of the Research Career, CONICET, Buenos Aires and ${ }^{\dagger}$ University of Mendoza, Mendoza, Argentina

Mailing address: Daniel Bia Santana, PhD, Physiology Department, School of Medicine, Republic University, General Flores 2125, Montevideo, Uruguay PC:11800. E-mail: dbia@ fmed.edu.uy

ISSN-1346-9843 doi:10.1253/circj.CJ-09-0496

All rights are reserved to the Japanese Circulation Society. For permissions, please e-mail: cj@j-circ.or.jp 
could differ (qualitatively and/or quantitatively) between elastic and muscular arteries. Although several in vitro studies of the adventitia function have been published, to our knowledge there are no in-vivo studies (a) evaluating the adventitial influence on the diameter and wall mechanics of peripheral muscular arteries or (b) analyzing the potential different active role of the adventitia in the peripheral muscular and central elastic arteries.

This study focused on (1) characterizing the geometric and mechanical properties of femoral arteries before and after the adventitia removal in both in-vivo and in-vitro experiments, and (2) a comparative analysis of the changes in central elastic arteries biomechanics associated with adventitia removal, using well-known indexes of arterial function. ${ }^{13-15}$ Additionally, as a secondary issue, we analyzed the existence of time-dependent biomechanical changes associated with adventitia removal. Taking into account our previous results, 4,16 we hypothesized that under in vivo conditions the adventitia would play a significant role (that could be even more important than that described in central elastic arteries) in determining basal geometric and mechanical properties in femoral arteries. Finally, because in our animal model physiological stability would be preserved, we expected compensatory mechanisms (ie, arterial wall shear stress-related) to modify the biomechanical changes associated with the adventitia removal.

\section{Methods}

The experimental protocol was approved by the Research and Development Council of the Biomedical Technology Department of the Favaloro University and the experiments conform to the "European Convention for the Protection of Vertebrate Animals used for Experimental and other Scientific Purposes" (Council of Europe No. 123, Strasbourg 1985).

\section{In Vivo Studies}

Seven healthy Corriedale sheep, weighting $30 \pm 2 \mathrm{~kg}$ and aged 12-16 months, were included. The experiment was performed under general anesthesia induced with sodium thiopental (20 mg/kg iv) and maintained with $1 \%$ halothane delivered through a Bain tube connected to a ventilator (Neumovent-910; Tecme S.A., Cordoba, Argentina). Respiratory parameters were monitored throughout the experiment. A pulse oximeter was used (Pulse Oximeter 515A, Novametrix Medical Systems Inc, Wallingford, CT, USA). Respiratory rate and tidal volume were checked and maintained in the physiological ranges.

A pressure sensor (Microtransducer, Micro Tip, SPC-3707F, Millar Instruments Inc, Houston, TX, USA) was positioned in the right iliac artery, near the origin of the femoral artery, via the brachiocephalic trunk. The sensor had been previously calibrated using a mercury manometer. Femoral artery diameter was measured using the ultrasonic technique routinely used in our laboratory. ${ }^{4,17}$ To this end, the right femoral artery was dissected at its origin, preserving the perivascular adipose tissue, and a pair of ultrasonic crystals ( $5 \mathrm{MHz}, 2-\mathrm{mm}$ diameter) was sutured to the vessel. Ultrasonic signals were visualized on an oscilloscope screen (Tektronix TOS-220, Tektronix Inc, Beaverton, OR USA). The transit time of the ultrasonic waves (velocity $=1,584 \mathrm{~m} / \mathrm{s}$ ) was converted into distance through a sonomicrometer (Triton Technology Inc, San Diego, CA, USA). Afterwards, the diameter signal was calibrated in millimeters using the sonomicrometer calibration facility. ${ }^{17}$ Cardiac output was measured using a Swan-Ganz catheter positioned in the pulmonary trunk via the right jugular vein. The flow stability was monitored with an ultrasonic flow-meter developed for animal use (Transonic Systems Inc, Ithaca, NY, USA).

Pressure and diameter recordings (sampling frequency= $500 \mathrm{~Hz}$ ) were performed $15 \mathrm{~s}$ before the adventitia was removed (preserving the perivascular adipose tissue), and 10 and $20 \mathrm{~min}$ after it was removed. In the sheep, adventitia removal is an easy procedure because its adherence to the media layer of the femoral arteries is weak. With the aid of a stereomicroscope, the perivascular fat, connective tissue and adventitia were gently dissected using forceps and scissors, during which the ultrasound crystals were removed and after the adventitia removal they were repositioned exactly in the previous site. ${ }^{4}$ At the end of the experiments, to evaluate the integrity of the media layer, the arterial segments were submitted to a histological examination.

After each in vivo experimental session, the animals were killed with an intravenous overdose of pentobarbital followed by potassium chloride. The correct position of the ultrasonic crystals was confirmed at necropsy. At the end of each experiment, a 6-cm length arterial segment (measured in vivo), was obtained and weighed, then submitted for histological analysis. ${ }^{4}$

\section{In Vitro Studies}

Seven healthy sheep of similar weights and ages to those studied in vivo were used in these experiments. During artery harvesting general anesthesia was induced and maintained with sodium thiopental $(20 \mathrm{mg} / \mathrm{kg}$, iv). All animals had mechanical respiratory assistance and respiratory parameters were maintained within the physiological limits.

The right femoral artery was exposed by dissection near its origin, preserving the perivascular adipose tissue, and then a solid-state pressure micro-transducer (Model P2.5, Konigsberg Instruments, Inc, Pasadena, CA, USA) was inserted through a collateral branch. Two miniature piezoelectric crystal transducers $(5 \mathrm{MHz}, 2-\mathrm{mm}$ in diameter) were sutured on opposite sides in the adventitia in order to measure the external diameter. Pressure and diameter calibration procedures were similar to those performed in the in vivo experiments. Two suture stitches were used to show a 6-cm length arterial segment, accurately measured with calipers. ${ }^{4,17,18}$

After sensor positioning, the pressure and diameter signals were visualized on the screen of a personal computer. The animal was killed with an intravenous overdose of sodium thiopental followed by potassium chloride and the selected femoral segment was excised and nontraumatically mounted at the in vivo length in an in vitro set-up (mock circulation) previously used by our group. ${ }^{4,19}$ After being placed in the specimen chamber, each arterial segment was allowed to equilibrate during 10 min under steady flow conditions $(450 \mathrm{ml} / \mathrm{min})$, stretching rate $(110$ beats $/ \mathrm{min})$ and mean pressure (approximately $85 \mathrm{mmHg}$ ). Flow stability was monitored with an ultrasonic flow-meter (Transonic Systems). During the in vitro experiments each arterial segment was kept immersed and perfused with thermally regulated $\left(37^{\circ} \mathrm{C}\right)$ and oxygenated Tyrode's solution $(\mathrm{pH} 7.4) .{ }^{4}$

After the equilibration period, pressure and diameter signals were recorded before and after adventitia removal (AAR), as in the in vivo experiments. The commands of the mock circulation were adjusted to reproduce in vivo wave morphology, enabling adequate isobaric, isoflow and isofrequency analysis. At the end of the experimental session, the segments were weighed and submitted to the histology laboratory. ${ }^{4}$ 
(A)

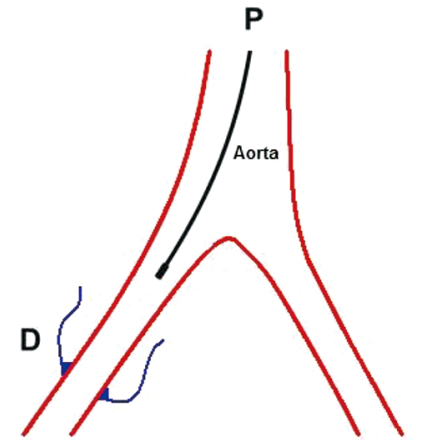

(C)

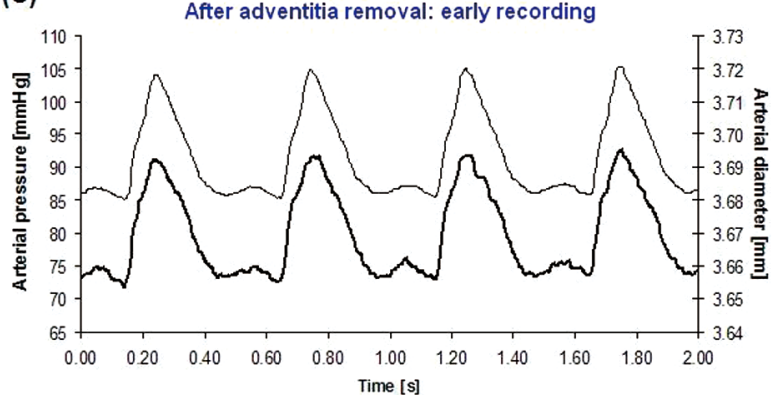

(B)

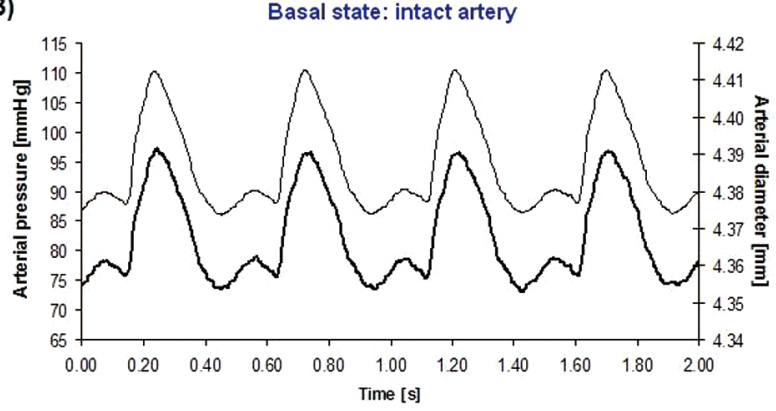

(D)

After adventitia removal: late recording

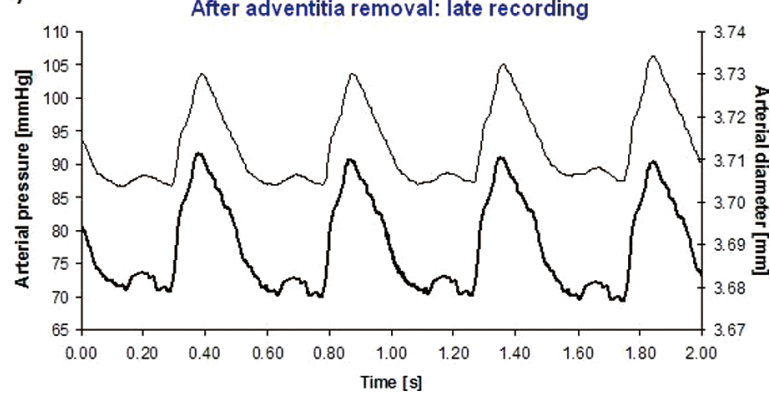

Figure 1. (A) Scheme of the in vivo instrumentation. D, ultrasonic microcrystals measuring femoral artery diameter; P, micro-tip solid-state pressure sensor. (B-D) Pressure (thin line) and diameter (thick line) recordings obtained during 3 different experimental states in a typical animal. (C, D) Recordings correspond to signals obtained 10 (early) and 20 (late) min after adventitia removal, respectively.

\section{Data Analysis}

To evaluate the arterial local biomechanical and functional behaviors, arterial compliance (AC), distensibility (AD), stiffness index $(\beta)$ and characteristic impedance $\left(\mathrm{Z}_{\mathrm{C}}\right)$ were calculated. ${ }^{20}$

The $\mathrm{AC}, \mathrm{AD}$ and $\beta$ are indexes commonly used in clinical practice because they can be obtained just by using systolic (maximal) and diastolic (minimal) pressure and diameter values: ${ }^{20}$

$\mathrm{AC}=\frac{(\mathrm{SD}-\mathrm{DD})}{(\mathrm{SBP}-\mathrm{DBP})}, \mathrm{AD}=\frac{(\mathrm{SD}-\mathrm{DD})}{(\mathrm{SBP}-\mathrm{DBP}) \mathrm{DD}}, \beta=\mathrm{In} \frac{\mathrm{SBP}}{\mathrm{DBP}} \frac{\mathrm{DD}}{(\mathrm{SD}-\mathrm{DD})}$

where SD and DD are the systolic and diastolic arterial external diameters, SBP and DBP are the systolic and diastolic blood pressures, and ln is the natural logarithm. The AC is a mechanical parameter that evaluates the arterial segment as a whole, and so it is influenced by both the arterial wall intrinsic stiffness and the geometric properties. ${ }^{20}$ In contrast, $\mathrm{AD}$ and $\beta$ provide information about arterial stiffness that is independent of arterial diameter, and so they enable comparison of arterial segments of different sizes. ${ }^{20}$ However, although $\mathrm{AD}$ describes the fractional pulsatile diameter changes that occur in an artery exposed to a given change in intraluminal pressure, assuming a linear pressure-diameter relationship, $\beta$ considers that the relationship can be fitted to a logarithmic function. ${ }^{20}$

The CF was evaluated by means of local hemodynamic impedance, quantified in terms of $\mathrm{Zc}_{\mathrm{c}}{ }^{4,18} \mathrm{An}$ increased $\mathrm{Zc}_{\mathrm{c}}$ determines augmented impedance against blood flow, resulting in a decreased capacity to conduct blood. Therefore, by inverse reasoning, $\mathrm{CF}$ was computed as $1 / \mathrm{Zc}_{\mathrm{c}}{ }^{18} \mathrm{Zc}$ was esti- mated using the Water-Hammer equation: ${ }^{20}$

$\mathrm{Z}_{\mathrm{C}}=\frac{\rho \cdot \mathrm{PWV}}{\mathrm{CSAD}}$

where $\rho$ is the blood density $\left(\rho=1.06 \mathrm{~g} / \mathrm{cm}^{3}\right)$, PWV is the pulse wave velocity estimated from the Moens-Korteweg equation and CSAD is the diastolic cross-sectional area. ${ }^{4,20}$

Finally, mean wall shear stress (WSS $\mathrm{m}$ ) was calculated:20

$\mathrm{WSS}_{\mathrm{m}}=\frac{32 \cdot \mathrm{AF} \cdot \mu}{\pi \cdot \mathrm{D}^{3}}$

where $\mathrm{AF}$ is the arterial blood flow level, $\mu$ is the viscosity (assumed to be $0.7 \times 10^{-2} \mathrm{dyn} \cdot \mathrm{s}^{-1} \cdot \mathrm{cm}^{-2}$ ) and $\mathrm{D}$ is the internal arterial diameter.

\section{Histologic Studies}

At the end of the in vivo and in vitro experiments, the arterial segments were submitted to histological examination to confirm the integrity of the media and intima layers and adequate adventitia removal. To analyze the samples under a light microscope the vascular sections were fixed in buffered $10 \%$ formaldehyde and embedded in paraffin, from which $5-\mu \mathrm{m}$-thick transverse sections were obtained and stained using hematoxylin-eosin, orcein and Cajal-Gallego stains. ${ }^{4}$

\section{Statistical Analysis}

Values are expressed as mean \pm standard deviation (MV \pm $\mathrm{SD})$. One-way analysis of variance and Bonferroni tests were used to compare in vivo data, because we compared 3 groups/conditions: (1) basal, (2) in vivo AAR Early, and (3) in vivo AAR Late. We had 2 in vitro groups: (1) in vitro 

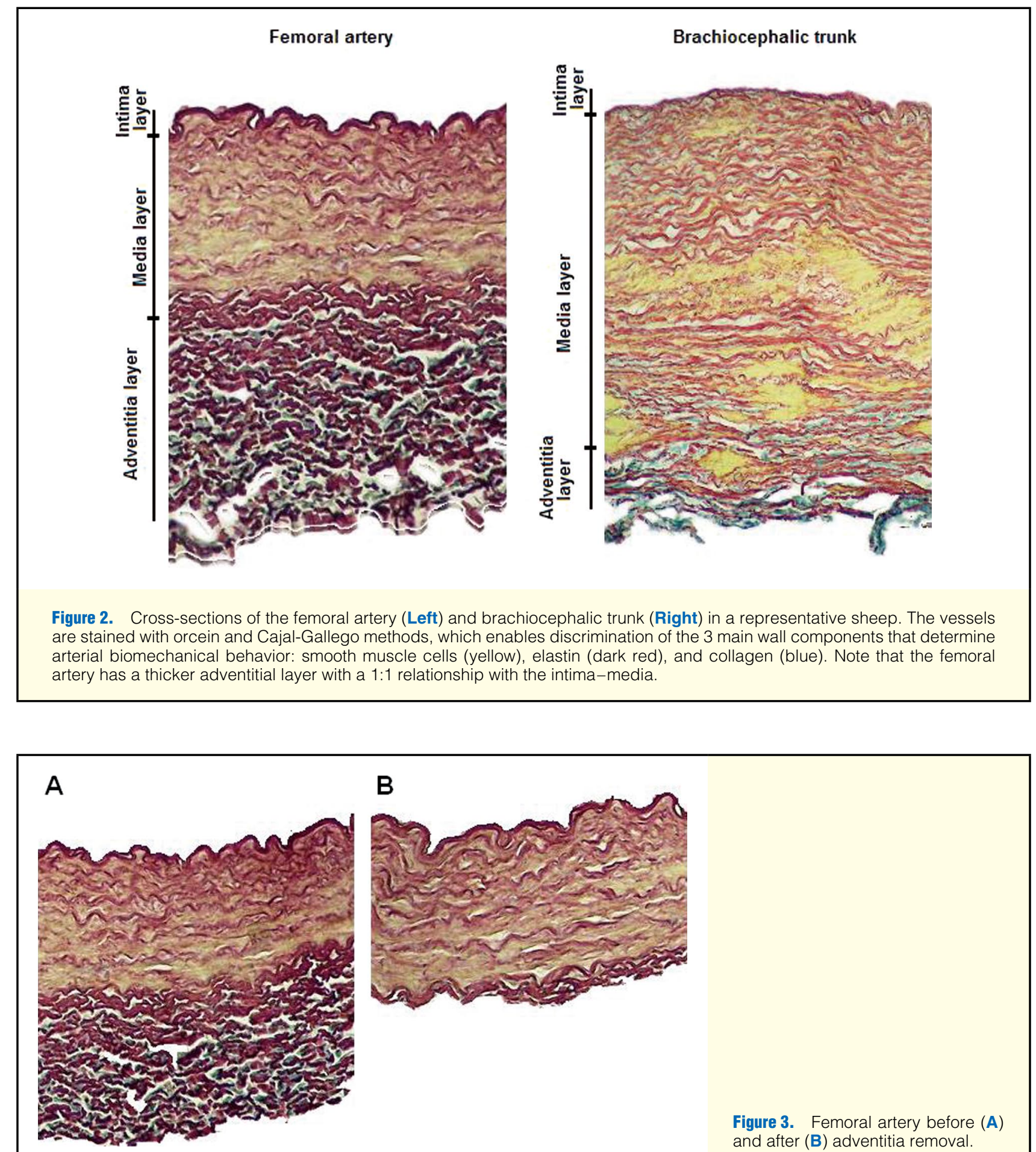

Figure 3. Femoral artery before (A) and after (B) adventitia removal.

intact artery (IA) and (2) in vitro AAR, so comparisons were done using t-test (unpaired, 2-tail). $\mathrm{P}<0.05$ was adopted as statistically significant.

From the pressure and diameter signals obtained in the brachiocephalic trunk, using an experimental methodology similar to the present one, ${ }^{4,16}$ the biomechanical parameters calculated for the femoral arteries were calculated for the brachiocephalic trunks, taking the isobaric and isofrequency conditions into consideration. Then, for each artery type (muscular and elastic), the effects of the adventitia removal on each parameter were quantified. The results were compared using t-test (unpaired, 2-tailed).

\section{Results}

No technical mistakes were observed in the experiments, neither related to surgical instrumentation (Figure 1A) nor during signal recordings before and AAR (Figures 1B-D).

Comparative observations during the experiments and after microscopic analysis showed that the adventitia of the 


\begin{tabular}{|c|c|c|c|c|c|c|c|}
\hline & \multirow{2}{*}{$\begin{array}{l}\text { In vivo IA } \\
M V_{ \pm S D}\end{array}$} & \multicolumn{3}{|c|}{ In vivo AAR (Early) } & \multicolumn{3}{|c|}{ In vivo AAR (Late) } \\
\hline & & $M V \pm S D$ & $\begin{array}{l}\Delta \% \text { with } \\
\text { respect to } \\
\text { in vivo IA }\end{array}$ & $\begin{array}{l}P \text { value with } \\
\text { respect to } \\
\text { in vivo IA }\end{array}$ & $M V \pm S D$ & $\begin{array}{l}\Delta \% \text { with } \\
\text { respect to } \\
\text { in vivo IA }\end{array}$ & $\begin{array}{l}P \text { value with } \\
\text { respect to } \\
\text { in vivo IA }\end{array}$ \\
\hline SBP $(\mathrm{mmHg})$ & $73.8 \pm 19.3$ & $74.5 \pm 16.7$ & 0.9 & 0.756 & $74.7 \pm 17.2$ & 1.1 & 0.690 \\
\hline $\mathrm{DBP}(\mathrm{mmHg})$ & $62.2 \pm 20.3$ & $61.3 \pm 16.6$ & -1.3 & 0.727 & $62.5 \pm 17.5$ & 0.5 & 0.870 \\
\hline $\mathrm{PP}(\mathrm{mmHg})$ & $11.7 \pm 2.5$ & $13.2 \pm 1.9$ & 12.9 & 0.017 & $12.2 \pm 3.1$ & 4.3 & 0.295 \\
\hline MBP (mmHg) & $66.1 \pm 20.0$ & $65.7 \pm 16.6$ & -0.5 & 0.884 & $66.6 \pm 17.3$ & 0.8 & 0.807 \\
\hline $\mathrm{SD}(\mathrm{mm})$ & $3.65 \pm 0.32$ & $3.39 \pm 0.19$ & -7.0 & 0.068 & $3.55 \pm 0.31$ & -2.6 & 0.574 \\
\hline $\mathrm{DD}(\mathrm{mm})$ & $3.49 \pm 0.33$ & $3.30 \pm 0.21$ & -5.4 & 0.131 & $3.46 \pm 0.33$ & -0.8 & 0.864 \\
\hline $\mathrm{MD}(\mathrm{mm})$ & $3.54 \pm 0.32$ & $3.33 \pm 0.20$ & -5.9 & 0.102 & $3.49 \pm 0.32$ & -1.4 & 0.757 \\
\hline $\mathrm{PD}(\mathrm{mm})$ & $0.16 \pm 0.09$ & $0.10 \pm 0.02$ & -41.2 & 0.077 & $0.09 \pm 0.03$ & -42.3 & 0.052 \\
\hline WSSm $\left(\mathrm{dyn} / \mathrm{cm}^{2}\right)$ & $12.05 \pm 1.45$ & $14.48 \pm 2.0$ & 20.1 & 0.040 & $12.58 \pm 1.71$ & 4.4 & 0.312 \\
\hline HR (beats/min) & $112.6 \pm 15.2$ & $111.2 \pm 12.2$ & -1.2 & 0.471 & $113.2 \pm 14.4$ & 0.6 & 0.751 \\
\hline $\mathrm{CO}(\mathrm{L} / \mathrm{min})$ & $2.7 \pm 0.8$ & $2.7 \pm 0.8$ & 0.1 & 0.350 & $2.7 \pm 0.8$ & 0.5 & 0.350 \\
\hline RR (breaths/min) & $10.9 \pm 1.0$ & $10.8 \pm 1.0$ & -1.1 & 0.350 & $10.6 \pm 1.1$ & -2.3 & 0.170 \\
\hline $\mathrm{pO}_{2}(\mathrm{mmHg})$ & $95.4 \pm 2.6$ & $95.5 \pm 2.7$ & 0.1 & 0.350 & $95.9 \pm 2.6$ & 0.5 & 0.350 \\
\hline $\mathrm{pCO}_{2}(\mathrm{mmHg})$ & $35.3 \pm 7.7$ & $35.1 \pm 7.8$ & -0.4 & 0.350 & $34.9 \pm 7.5$ & -1.1 & 0.197 \\
\hline
\end{tabular}

Data $(n=7)$ are expressed as mean value $(M V) \pm$ standard deviation (SD). Statistical analysis, 1-way analysis of variance (ANOVA) and Bonferroni tests.

IA, intact artery; AAR, after adventitial removal; AAR Early and AAR Late, recordings done after 10 and 20 min, respectively; SBP, DBP, PP and MBP, systolic, diastolic, pulse and mean blood pressure, respectively; SD, DD, MD and PD, systolic, diastolic, mean and pulsatile diameter, respectively; WSSm, mean wall shear stress; HR, heart rate; CO, cardiac output; RR, respiratory rate.

femoral arteries was thicker than that of the brachiocephalic arteries (Figure 2). In all cases, AAR, the integrity of both the media and intima was preserved (Figure 3).

\section{Effects of Adventitia Removal}

In Vivo Experiments Cardiac output, mean systolic and diastolic pressures, and the heart and respiratory rates remained unchanged throughout the experimental session, confirming the experimental model's stability (Table 1). The $\mathrm{pO}_{2}$ and $\mathrm{pCO}_{2}$ values behaved similarly. A significant increase in pulse pressure was observed early AAR $(\mathrm{P}<0.05)$. A nonsignificant decrease in the arterial diameter (systolic, diastolic, mean and pulsate) was observed in both early and late recordings AAR (Table 1). When the wall shear stress was analyzed the results showed an early increase $(\mathrm{P}<0.05)$ followed by a reduction (late recordings), reaching levels similar to the basal conditions (Table 1).

In all the animals, for each cardiac cycle, there was off-line visualization of the femoral artery instantaneous pressurediameter loop. A leftward shift was observed early (10 min) AAR (Figure 4). At $20 \mathrm{~min}$ after removing the adventitia (late recordings), there was a rightward shift of the pressurediameter loop (Figure 4). Nevertheless, in all cases, the IA loops remained at the right side of the loops corresponding to the AAR.

Biomechanical analysis of the femoral arteries using parameters that consider the vessel as a whole structure and the arterial wall biomechanics (ie, $\mathrm{AC}, \mathrm{AD}, \beta$ ) showed an increase in stiffness in both the early and late recordings AAR (Table 2). When $\mathrm{CF}$ was analyzed the results showed an early reduction $(\mathrm{P}<0.02)$ followed by a nonsignificant reduction in the late recordings (Table 2).

In Vivo vs In Vitro Experiments The isobaric, isofrequency and isoflow comparisons between the in vivo and in vitro IA showed higher arterial stiffness under in vitro conditions. Additionally, despite the higher stiffness found in vitro in intact femoral arteries, the larger diameter values found in these arteries determined an increase in $\mathrm{CF}(\mathrm{P}<0.002)$ (Table 3).

AAR in vitro, nonsignificant changes were observed in mean wall shear-stress and biomechanical properties (isobaric, isofrequency and isoflow analyses), despite an increase in arterial diameter $(\mathrm{P}<0.002)$ (Table 3, Figure 4).

Muscular vs Elastic Arteries The isobaric and isofrequency changes in $\mathrm{AC}, \mathrm{AD}$ and $\beta$ associated with adventitia removal were higher in muscular arteries (Table 4). In contrast, in relative terms, the diameter reduction was higher in central elastic vessels, determining a major $\mathrm{CF}$ reduction in these arteries AAR (Table 4).

\section{Discussion}

\section{Physiological Considerations}

The work main findings can be summarized as 3 points: biomechanics, arterial diameter and $\mathrm{CF}$, and temporal changes.

Biomechanics First, AAR in vivo, but not in vitro, the muscular arteries' biomechanical properties and conduit capability changed. In particular, adventitia removal was associated with an increase in arterial stiffness, a decrease in the conduit capability and a nonsignificant, but relevant, reduction in arterial diameter. These results suggest that the changes in the femoral arteries AAR could be ascribed to smooth muscle-dependent mechanisms.

To understand these results some practical issues should be considered. In both the in vivo and in vitro studies the arterial diameter and pressure were assessed before and after mechanical removal of the adventitia. The in vivo experiments enabled characterization of the adventitial function in states with preserved smooth muscle tone and reactivity (capable of modifying the activation level). Hence, if the adventitia had a role in smooth muscle tone regulation it would be evidenced during the in vivo studies. However, the adventitia's biomechanical role in vivo could depend on active (ie, smooth muscle-dependent), but also on recognized 


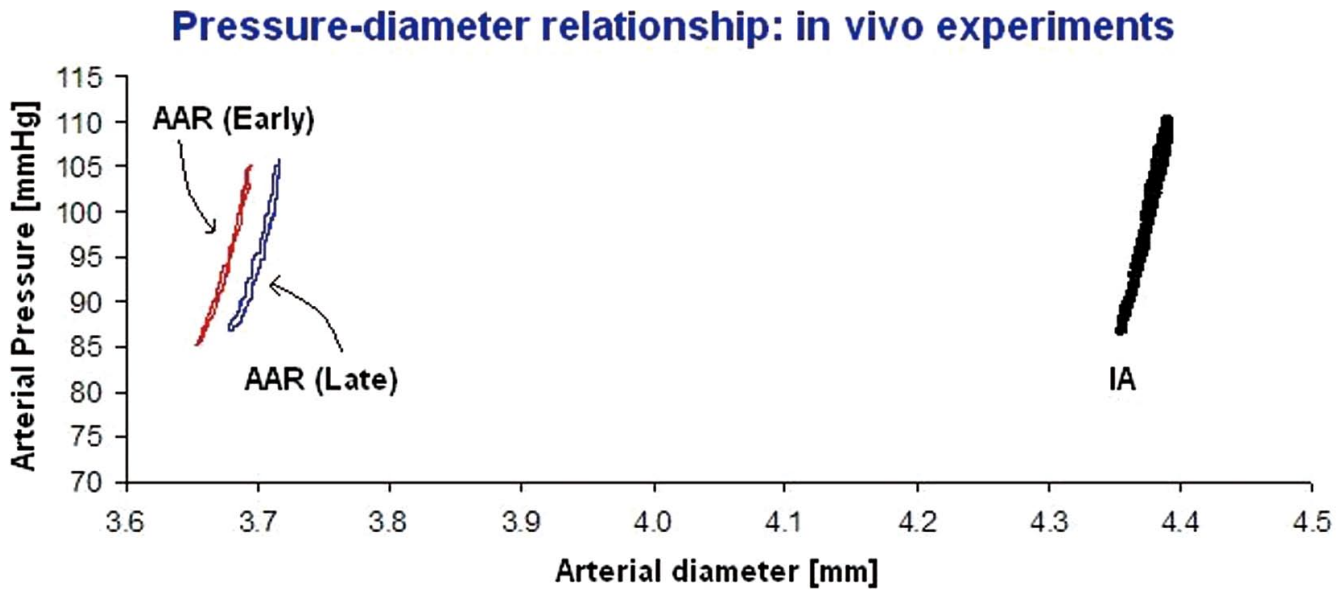

Pressure-diameter relationship: in vitro experiments

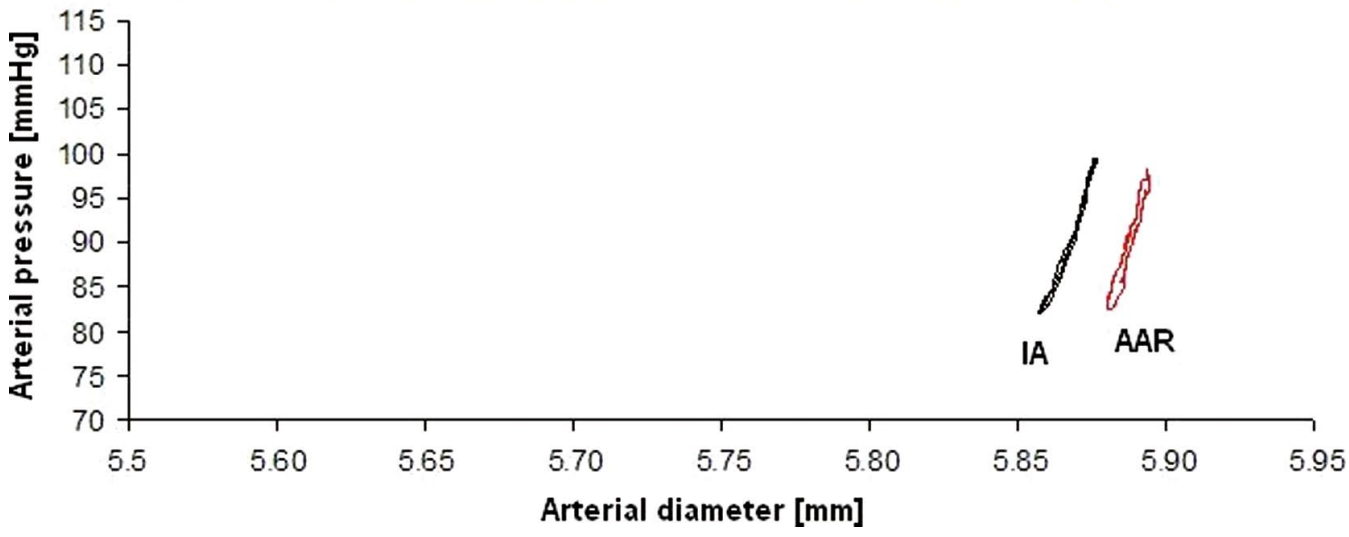

Figure 4. (Upper) In vivo pressure-diameter instantaneous relationship obtained in an intact femoral artery (IA; black pressure-diameter loop), and 10 min (early recording; red pressure-diameter loop) and 20 min (late recording; blue pressurediameter loop) after adventitia removal (AAR). (Lower) In vitro pressure-diameter instantaneous relationship obtained both in an IA (black pressure-diameter loop) and AAR (red pressure-diameter loop). Note that the left shift in the in vivo pressurediameter loop (diameter contraction) is not present in the in vitro studies.

\begin{tabular}{|c|c|c|c|c|c|c|c|}
\hline & \multirow{2}{*}{$\begin{array}{l}\text { In vivo IA } \\
M \mathrm{MV}_{ \pm} \mathrm{SD}\end{array}$} & \multicolumn{3}{|c|}{ In vivo AAR (Early) } & \multicolumn{3}{|c|}{ In vivo AAR (Late) } \\
\hline & & $M V \pm S D$ & $\begin{array}{c}\Delta \% \text { with } \\
\text { respect to } \\
\text { in vivo IA }\end{array}$ & $\begin{array}{l}\text { P value with } \\
\text { respect to } \\
\text { in vivo IA }\end{array}$ & $M V \pm S D$ & $\begin{array}{l}\Delta \% \text { with } \\
\text { respect to } \\
\text { in vivo IA }\end{array}$ & $\begin{array}{l}P \text { value with } \\
\text { respect to } \\
\text { in vivo IA }\end{array}$ \\
\hline Compliance $\left(10^{-3} \mathrm{~mm} / \mathrm{mmHg}\right)$ & $13.63 \pm 5.76$ & $7.38 \pm 2.16$ & -45.6 & 0.026 & $7.79 \pm 2.05$ & -42.5 & 0.034 \\
\hline Distensibility $\left(10^{-3} / \mathrm{mmHg}\right)$ & $3.99 \pm 1.87$ & $2.27 \pm 0.74$ & -43.0 & 0.041 & $2.27 \pm 0.62$ & -43.1 & 0.039 \\
\hline$\beta$-index & $4.59 \pm 1.94$ & $7.50 \pm 2.64$ & 63.4 & 0.007 & $7.09 \pm 1.79$ & 54.5 & 0.018 \\
\hline $\mathrm{CF}\left(10^{-2} \mathrm{dyn} \cdot \mathrm{s}^{-1} \cdot \mathrm{cm}^{-5}\right)$ & $1.80 \pm 0.34$ & $1.31 \pm 0.09$ & -27.0 & 0.016 & $1.47 \pm 0.29$ & -18.3 & 0.128 \\
\hline
\end{tabular}

Data $(n=7)$ are expressed as MV \pm SD. Statistical analysis, 1-way ANOVA and Bonferroni tests.

$\mathrm{CF}$, conduit function. Other abbreviations see in Table 1.

structural or passive mechanisms. Therefore, to discriminate the contribution of passive and active mechanisms, we performed the in vitro studies, in which active mechanisms were minimized or blocked. The integrated analysis of the in vitro and in vivo data allowed us to characterize the adventitial role in arterial biomechanics.

As stated, adventitia removal in the in vivo studies resulted in (a) nonsignificant diameter reduction, (b) increased arte- rial stiffness and (c) reduction in CF (Tables 1,2, Figure 4). The arterial diameter reduction observed AAR supports an active mechanism of control by the adventitia, because the opposite response would be expected if there were an adventitial-dependent restriction of arterial dilatation. The arterial changes observed AAR could be mediated only by a smooth muscle contraction. Additionally, this is in agreement with the finding of increased wall viscosity (which depends on the 


\begin{tabular}{|c|c|c|c|c|c|c|}
\hline & \multirow{2}{*}{$\begin{array}{c}\text { In vitro IA } \\
M V \pm S D\end{array}$} & \multicolumn{3}{|c|}{ In vitro AAR } & \multicolumn{2}{|c|}{ In vivo IA vs In vitro IA } \\
\hline & & $\mathrm{MV} \pm \mathrm{SD}$ & $\begin{array}{l}\Delta \% \text { with } \\
\text { respect to } \\
\text { in vitro IA }\end{array}$ & $\begin{array}{l}P \text { value with } \\
\text { respect to } \\
\text { in vitro IA }\end{array}$ & $\begin{array}{l}\Delta \% \text { with } \\
\text { respect to } \\
\text { in vivo IA }\end{array}$ & $\begin{array}{l}P \text { value with } \\
\text { respect to } \\
\text { in vivo IA }\end{array}$ \\
\hline $\mathrm{SBP}(\mathrm{mmHg})$ & $82.4 \pm 2.6$ & $82.9 \pm 3.1$ & 0.5 & 0.722 & 11.6 & 0.265 \\
\hline $\mathrm{DBP}(\mathrm{mmHg})$ & $66.7 \pm 2.9$ & $66.8 \pm 2.9$ & 0.1 & 0.951 & 7.4 & 0.564 \\
\hline $\mathrm{PP}(\mathrm{mmHg})$ & $15.7 \pm 4.4$ & $16.0 \pm 3.1$ & 2.2 & 0.864 & 34.4 & 0.864 \\
\hline MBP (mmHg) & $72.0 \pm 1.8$ & $72.2 \pm 2.5$ & 0.3 & 0.803 & 9.0 & 0.448 \\
\hline $\mathrm{SD}(\mathrm{mm})$ & $5.79 \pm 0.25$ & $5.85 \pm 0.24$ & 1.0 & 0.001 & 58.8 & 0.001 \\
\hline $\mathrm{DD}(\mathrm{mm})$ & $5.71 \pm 0.25$ & $5.76 \pm 0.25$ & 1.0 & 0.001 & 63.7 & 0.001 \\
\hline $\mathrm{MD}(\mathrm{mm})$ & $5.73 \pm 0.25$ & $5.79 \pm 0.25$ & 1.0 & 0.001 & 62.0 & 0.001 \\
\hline $\mathrm{PD}(\mathrm{mm})$ & $0.08 \pm 0.02$ & $0.09 \pm 0.02$ & 1.5 & 0.811 & -47.8 & 0.041 \\
\hline WSSm $($ dyn/cm²) & $2.84 \pm 0.27$ & $2.76 \pm 0.24$ & -3.1 & 0.843 & -77.1 & 0.001 \\
\hline HR (beats/min) & $109.7 \pm 0.9$ & $109.5 \pm 0.9$ & -0.2 & 0.699 & -2.5 & 0.632 \\
\hline Compliance $\left(10^{-3} \mathrm{~mm} / \mathrm{mmHg}\right)$ & $5.61 \pm 1.67$ & $5.36 \pm 1.34$ & -4.4 & 0.788 & -58.6 & 0.004 \\
\hline Distensibility $\left(10^{-4} / \mathrm{mmHg}\right)$ & $9.80 \pm 2.62$ & $9.36 \pm 2.57$ & -4.5 & 0.780 & -75.4 & 0.001 \\
\hline$\beta$-index & $14.44 \pm 3.13$ & $15.18 \pm 3.67$ & 5.2 & 0.692 & 214.8 & 0.001 \\
\hline CF $\left(10^{-2} \mathrm{dyn} / \mathrm{cm}^{5}\right)$ & $2.72 \pm 0.49$ & $2.69 \pm 0.27$ & -1.0 & 0.714 & 51.3 & 0.001 \\
\hline
\end{tabular}

Data $(n=7)$ are expressed as $M V \pm S D$. Statistical analysis, t-test.

Abbreviations see in Table 1.

\begin{tabular}{|c|c|c|}
\hline & $\begin{array}{l}\text { Femoral artery (Muscular artery) } \\
\qquad n=7\end{array}$ & $\begin{array}{l}\text { Brachiocephalic trunk (Elastic artery) } \\
\qquad n=6\end{array}$ \\
\hline Compliance & $-45.6 \pm 8.7$ & $-34.1 \pm 6.7^{\star}$ \\
\hline Distensibility & $-43.0 \pm 9.3$ & $-26.8 \pm 7.6^{*}$ \\
\hline$\beta$-index & $63.4 \pm 12.1$ & $36.5 \pm 7.8^{*}$ \\
\hline Mean arterial diameter & $-5.9 \pm 1.2$ & $-14.1 \pm 4.5^{*}$ \\
\hline $\mathrm{CF}$ & $-27.0 \pm 7.5$ & $-44.2 \pm 8.2^{*}$ \\
\hline
\end{tabular}

Data are $\mathrm{MV} \pm \mathrm{SD}$. Statistical analysis: t-test. ${ }^{*} \mathrm{P}-0.05$ with respect to the change observed in femoral arteries.

Abbreviations see in Tables 1,2.

level of smooth muscle tone) in elastic arteries AAR.4,11,16,17 Similar to Stefanadis et al, who found a reduction in aortic distensibility after removing the adventitia, ${ }^{21}$ we found a reduction in femoral distensibility AAR, which also suggests that our in vivo results depended on smooth muscle activity, because under physiological pressures the arterial mechanical properties mainly depend on the elastin fibers and smooth muscle, and smooth muscle activation would decrease compliance and distensibility. ${ }^{11,20}$ Finally, the in vivo/in vitro comparisons also support that smooth muscle mediates the adventitial control role, because no adventitial-dependent mechanical changes were obtained in vitro (Table 3, Figure 4).

That the adventitial effects on arterial biomechanics are active (related to smooth muscle tone) rather than passive was also suggested by Schulze-Bauer et al, ${ }^{22}$ who authors showed that adventitial compliance is high at low pressure levels and that as the pressure increases marked stiffening occurs. Hence, the passive or structural role of the adventitia on biomechanical behavior would increase as pressure rises. The high compliance or distensibility of the adventitia at physiological pressures could also explain why we did not find an increase in compliance and distensibility, despite removing a wall lamina (ie, the adventitia) during the in vitro experiments.
In agreement with our findings, several authors describe the existence of adventitial and perivascular fat relaxing factors involved in arterial basal tone and reactivity regulation. ${ }^{5,23,24}$ The finding of an acute and active arterial contraction associated with the removal of the adventitia and perivascular fat could reasonably be expected. Additionally, it is noteworthy that when removing the adventitia and perivascular adipose tissue, nerve terminals that may release vasoactive factors are also removed. So, the geometric and biomechanical changes could be ascribed to the removal of the adventitia, perivascular adipose tissue and/or nerve ends.

Differences between in vitro and in vivo experiments have been previously reported by our group ${ }^{4}$ and comparing in vitro and in vivo studies before adventitia removal was not an aim of this work. However, it is noteworthy that, in agreement with the present experimental results, we previously described an arterial diameter and stiffness increase after vascular excision, which could be ascribed to a reduction in smooth muscle tone and reactivity under in vitro conditions. ${ }^{4}$

Arterial Diameter and CF Second, in muscular arteries the changes in $\mathrm{AC}, \mathrm{AD}$ and $\beta$ associated with adventitia removal were greater than those found in elastic arteries. In contrast, AAR the changes in arterial diameter and CF were higher in central elastic arteries (Table 4).

The thickness of the adventitial layer varies according to 
the type and location of the artery. ${ }^{25}$ In dogs the adventitia of elastic arteries is relatively thin, constituting in general terms only $10 \%$ of the vascular wall thickness, whereas in muscular arteries the adventitia often represents half of the vascular wall. ${ }^{24}$ These observations were confirmed in our study of ovine arteries (Figure 2).

The functional relevance of the structural regional differences between the adventitia of elastic and muscular arteries is poorly recognized. From a functional point of view it could be hypothesized that the thicker adventitia found in peripheral muscular arteries could contribute "passively" to avoiding the pressure-dependent major distension that could occur in these vessels as a result of the central-to-peripheral pulse pressure amplification. However, in the light of our results it could be proposed that there are not only "passive" differences in the adventitia's role in central elastic and in peripheral muscular arteries. In this regard, in peripheral muscular arteries the adventitia could play a major role in determining the basal biomechanical properties (ie, compliance), while in the central elastic arteries the adventitia would mainly regulate arterial impedance and so the cardiac dynamic afterload (Table 4). In other words, the adventitia could affect arterial properties through passive and active mechanisms, with quantitatively different effects on central and peripheral vessels.

Temporal Changes Third, there were time-dependent differences in the biomechanical changes observed after removing the adventitia in vivo. The initial major changes in the arterial biomechanical properties, associated with adventitia removal, were subsequently reduced. However, the biomechanical values stabilized at new levels, yet different with respect to those found in the basal state (intact arteries).

This is the first study describing a time-dependence in the changes associated with adventitia removal. In vivo, adventitia removal initially $(10 \mathrm{~min})$ resulted in arterial constriction, but afterward $(20 \mathrm{~min})$ there was a gradual vasodilator response (Table 1, Figure 4). Additionally, early AAR there was a great reduction in $\mathrm{CF}$ and an increase in arterial stiffness (ie, $\beta$ ), but $20 \mathrm{~min}$ after adventitial removal the described parameters reached new levels more like those in the basal condition (Table 2).

To determine the mechanisms that explain the time-dependence of the changes in biomechanical properties AAR was beyond the scope of the present study. However, at least in theory, some aspects should be considered.

First, our findings could be partially explained by the described adventitia-endothelium interaction and counterbalance. ${ }^{6,26}$ The early changes in arterial geometry (diameter) and wall biomechanics associated with adventitia removal could be mediated by a sudden imbalance between endothelium-mediated and adventitia-mediated effects on the smooth muscle cells localized in the media layer. Adventitia removal could result in a distortion in the equilibrium between vasoconstrictors and vasorelaxing agents, causing a sudden tendency to vasoconstriction. Then, minutes AAR, a wall compensatory adaptation would be expected (ie, increased production and release of endothelium-relaxing factors because of the increase in mean wall shear-stress) (Table 1). In this way, a new state is reached in which mean wall shear stress levels are similar to those in basal conditions, an explanation that is in agreement with the concept that shear-stress levels are controlled. ${ }^{20}$

In agreement with that statement above, AAR there are reported changes in nitric oxide release and half-life. Nevertheless, it is noteworthy that several regulatory factors (ie, neuropeptide $\mathrm{Y}$, substance $\mathrm{P}$ ) are present in the adventitia and could also contribute to the smooth muscle and time-dependent biomechanical changes associated with its removal. ${ }^{2}$

Methodological Considerations: Strengths and Limitations To understand our results some methodological and experimental issues should be considered. First, taking into account that the arterial wall properties are pressure-, frequencyand flow-dependent, the in vivo and in vitro studies were performed under isobaric, isoflow and isofrequency conditions ${ }^{11,17}$ (Tables 1,3, Figure 4). Second, characterization of arterial properties was performed using well-known indexes previously used to evaluate both functional and structural vascular wall constituents. ${ }^{27,28}$

The main limitation of our study is technical, because the reduced lumen of the femoral artery meant we could not place the pressure solid catheter next to the ultrasonic crystals. Hence, we could not calculate the arterial viscous modulus as we did previously. ${ }^{4}$ Besides, during the recordings we could not place a flow-meter in the femoral artery because placement of the probe could have created a "cuff effect", situation reported when the blood flow of the first-order vasa vasorum is impaired with a silastic collar positioned around the artery, leading to an arterial wall hypoxia. ${ }^{29}$ However, before pressure and diameter recordings, the femoral blood flow was measured each of the in vivo and in vitro experiments to reproduce similar conditions.

\section{Clinical Relevance}

Our findings have clinical implications. In some surgical procedures (eg, arterial bypass), to make the anastomotic junction with the vascular substitute easier, the adventitia of the recipient native vessel is removed. Additionally, when veins (ie, human great saphenous vein in lower limb revascularization) and/or arteries (ie, radial or mammary arteries in myocardial revascularization, iliofemoral arteries cryopreserved to be used in lower limb revascularization) are procured to be used as vascular substitutes the adventitia layer is frequently disrupted. Taking into account our results, at least in theory, this could have negative effects on vascular biomechanics, and consequently could result in negative changes in the performance of the vascular substitute (ie, increased stiffness). On this point, in a study performed to improve venous graft patency, 3 harvesting techniques were compared and it was concluded that preserving the saphenous vein endothelium, adventitia and surrounding tissue, using a "notouch" technique, abolished intraoperative venospasm and resulting in improved graft patency. ${ }^{30}$ In addition, Dashwood et al reported that perivascular fat-derived nitric oxide had a beneficial role in vein grafts that had been atraumatically harvested. ${ }^{31}$ Finally, Souza et $\mathrm{al}^{32}$ demonstrated that harvesting saphenous vein to be used in coronary bypass with its intact adventitia and surrounding tissues was associated with high short- and long-term patency rates of the venous grafts. Consequently, nowadays several authors propose the use of a "non-touch" technique during venous procurement to avoid (a) adventitial disruption that occurs when conventional harvesting techniques are used, and (b) the detrimental changes on the venous graft's function. Our results give complementary data, suggesting a physiological explanation for the problem found in clinical practice.

On the other hand, some therapeutic procedures determine changes in the adventitia. For instance, arterial angioplasty is associated with a stretching and splitting of the intima, media and adventitia, as well as of the vasa vasorum endothelium. This would trigger an impairment of the endothe- 
lium-dependent control of the arterial wall blood flow and could determine changes in wall dynamics. ${ }^{29}$ Hence, looking at our results, adventitial changes associated with angioplasty could determine an increase in arterial stiffness and a reduction in conduit capability.

In summary, femoral artery wall stiffness and $\mathrm{CF}$ were altered in our in vivo studies AAR. The observed changes were time-dependent and could be mediated by active regulation of arterial smooth muscle tone. The changes found in femoral artery compliance, distensibiliy and stiffness index were qualitatively similar, but quantitatively higher than those observed in the ovine brachiocephalic trunk (a central elastic artery). In contrast, the changes observed in diameter and CF were higher in the brachiocephalic trunk. These study results add evidence to the remarkable role of the adventitial layer in the physiological regulation of arterial wall dynamics.

\section{Acknowledgments}

This work was supported by the Favaloro University and funds of "BID 1728/OC-AR-PICTO-2004-20797" (Argentina), and PEDECIBA and ANII-FCE-2007-635 (Dr Armentano) and ANII-FCE-2007-638 (Dr Bia, Uruguay).

The authors thank Mr Juan Fernández and Ms Paula Bia Santana for their contribution to the histological studies and images processing.

\section{Disclosure}

Conflict of interest: none.

\section{References}

1. Rey E, Pagano P. The reactive adventitia: Fibroblast oxidase in vascular function. Arterioscler Thromb Vasc Biol 2002; 22: 1962 1971.

2. Auger FA, D'Orleans-Juste P, Germain L. Adventitia contribution to vascular contraction: Hints provided by tissue-engineered substitutes. Cardiovasc Res 2007; 75: 669-678.

3. Bia D, Zócalo Y, Pessana F, Armentano R, Pérez H, Saldías M, et al. Differential functional coupling between human saphenous cryoallografts and arteries: Importance of the arterial type and the biomechanical parameter evaluated. Artif Organs 2007; 31: 809-818.

4. Cabrera-Fischer E, Bia D, Camus J, Zócalo Y, De Forteza E, Armentano R. Adventitia-dependent mechanical properties of brachiocephalic ovine arteries in in vivo and in vitro studies. Acta Physiol 2006; 188: $103-111$.

5. Löhn M, Dubrovska G, Lauterbach B, Luft FC, Gollasch M, Sharma AM. Periadventitial fat releases a vascular relaxing factor. FASEB J 2002; 16: $1057-1063$.

6. Somoza B, González MC, González JM, Abderrahim F, Arribas SM, Fernández-Alfonso MS. Modulatory role of the adventitia on noradrenaline and angiotensin II responses: Role of endothelium and $\mathrm{AT}_{2}$ receptors. Cardiovasc Res 2005; 65: 478-486.

7. Okada M, Hasebe N, Aizawa Y, Izawa K, Kawabe J, Kikuchi K. Thermal treatment attenuates neointimal thickening with enhanced expression of heat-shock protein 72 and suppression of oxidative stress. Circulation 2004; 109: 1763-1768.

8. Gálvez-Prieto B, Dubrovska G, Cano MV, Delgado M, Aranguez I, González MC, et al. A reduction in the amount and anti-contractile effect of periadventitial mesenteric adipose tissue precedes hypertension development in spontaneously hypertensive rats. Hypertens Res 2008; 31: 1415-1423.

9. Cai WJ, Koltai S, Kocsis E, Scholz D, Kostin S, Luo X, et al. Remodeling of the adventitia during coronary arteriogenesis. Am J Physiol Heart Circ Physiol 2003; 284: H31 - H40.

10. Arribas SM, Daly CJ, González MC, Mc Grath JC. Imaging the vascular wall using confocal microscopy. J Physiol 2007; 584: 5 9.

11. Bia D, Barra JG, Grignola JC, Ginés FF, Armentano RL. Pulmonary artery smooth muscle activation attenuates arterial dysfunction during acute pulmonary hypertension. J Appl Physiol 2005; 98: $605-613$

12. Zócalo Y, Pessana F, Bia D, Armentano RL. Regional differences in vein wall dynamics under arterial hemodynamic conditions: Comparison with arteries. Artificial Organs 2006; 30: 265-275.

13. Agata J, Nagahara D, Kinoshita S, Takagawa Y, Moniwa N, Yoshida $\mathrm{D}$, et al. Angiotensin II receptor blocker prevents increased arterial stiffness in patients with essential hypertension. Circ J 2004; 68: 1194-1198.

14. Park SM, Seo HS, Lim HE, Shin SH, Park CG, Oh DJ, et al. Assessment of arterial stiffness index as a clinical parameter for atherosclerotic coronary artery disease. Circ J 2005; 69: 1218-1222.

15. Meguro T, Nagatomo Y, Nagae A, Seki C, Kondou N, Shibata M, et al. Elevated arterial stiffness evaluated by brachial-ankle pulse wave velocity is deleterious for the prognosis of patients with heart failure. Circ J 2009; 73: 673-680.

16. Bia D, Zócalo Y, Armentano RL, Camus J, De Forteza E, CabreraFischer EI. The adventitia reduces left ventricular dynamic afterload via smooth muscle activation-dependent mechanisms. Rev Esp Cardiol 2007; 60: 501-509.

17. Bia D, Armentano R, Craiem D, Grignola J, Ginés F, Simon A, et al. Smooth muscle role on pulmonary arterial function during acute pulmonary hypertension in sheep. Acta Physiol Scand 2004; 181: 359-366.

18. Bia D, Aguirre I, Zócalo Y, Devera L, Cabrera-Fischer E, Armentano R. Regional differences in viscosity, elasticity and wall buffering function in systemic arteries: Pulse wave analysis of the arterial pressure-diameter relationship. Rev Esp Cardiol 2005; 58: 167-174.

19. Cabrera-Fischer EI, Armentano RL, Pessana FM, Graf S, Romero L, Christen A, et al. Endothelium-dependent arterial wall tone elasticity modulated by blood viscosity. Am J Physiol Heart Circ Physiol 2002; 282: H389-H394.

20. Nichols W, O'Rourke M. Properties of the arterial wall. In: Nichols W, O'Rourke M, editors. McDonald's blood flow in arteries: Theoretical, experimental and clinical principles, $4^{\text {th }}$ edn. London, UK: Edward Arnold, 2005; 49-65, 67-93.

21. Stefanadis C, Vlachopoulus C, Karayannacos P, Boudoulas H, Stratos C, Filippides T, et al. Effect of vasa vasorum flow on structure and function of the aorta in experimental animals. Circulation 1995; 91: 2669-2678.

22. Schulze-Bauer CA, Regitnig P, Holzapfel GA. Mechanics of the human femoral adventitia including the high-pressure response. Am J Physiol Heart Circ Physiol 2002; 282: H2427-H2440.

23. Gutterman D. Adventitia-dependent influences on vascular function. Am J Physiol 1999; 277: H1265-H1272.

24. Verlohren S, Dubrovska G, Tsang S, Essin K, Luft FC, Huang Y, et al. Visceral periadventitial adipose tissue regulates arterial tone of mesenteric arteries. Hypertension 2004; 44: 271-276.

25. Rhodin J. Architecture of the vessel wall. In: Bohr D, Somlyo A, Sparks H, editors. Handbook of physiology: The cardiovascular system, Vol. II. Bethesda, MD: American Physiology Society, 1980; $1-31$.

26. González M, Arribas S, Molero F, Fernández-Alfonso M. Effect of removal of adventitia on vascular smooth muscle contraction and relaxation. Am J Physiol Heart Circ Physiol 2001; 280: H2876$\mathrm{H} 2881$.

27. Tuzun N, Tanriverdi H, Evrengul H, Kuru DS, Ergene AO. Aortic elastic properties in patients with coronary artery ectasia. Circ $J$ 2007; 71: 506-510.

28. Hongo M, Tsutsui H, Mawatari E, Hidaka H, Kumazaki S, Yazaki $\mathrm{Y}$, et al. Fluvastatin improves arterial stiffness in patients with coronary artery disease and hyperlipidemia: A 5-year follow-up study. Circ J 2008; 72: 722-728.

29. Scotland R, Vallance P, Ahluwalia A. Endogenous factors involved in regulation of tone of arterial vasa vasorum: Implications for conduit vessel physiology. Cardiovasc Res 2002; 46: 403-411.

30. Souza DS, Dashwood MR, Tsui JC, Filbey D, Bodin L, Johansson $\mathrm{B}$, et al. Improved patency in vein grafts harvested with surrounding tissue: Results of a randomized study using three harvesting techniques. Ann Thorac Surg 2002; 73: 1189-1195.

31. Dashwood MR, Dooley A, Shi-Wen X, Abraham DJ, Souza DSR. Does periadventitial fat-derived nitric oxide play a role in improved saphenous vein graft patency in patients undergoing coronary artery bypass surgery? J Vasc Res 2007; 44: 175-181.

32. Souza D, Johansson B, Bojö L, Karlsson R, Geijer H, Filbey D, et al. Harvesting the saphenous vein with surrounding tissue for CABG provides long-term graft patency comparable to the left internal thoracic artery: Results of a randomized longitudinal trial. J Thorac Cardiovasc Surg 2006; 132: 373-378. 